\title{
Automatic radiotherapy plan emulation for 3D dose reconstruction to enable big data analysis for historically treated patients
}

\author{
Ziyuan Wang*a, Marco Virgolin ${ }^{\mathrm{b}}$, Peter A.N. Bosman ${ }^{\mathrm{b}}$, Brian V. Balgobind ${ }^{\mathrm{a}}$, Arjan Bel ${ }^{\mathrm{a}}$, Tanja \\ Alderliesten $^{\mathrm{a}}$ \\ aDepartment of Radiation Oncology, Amsterdam UMC, University of Amsterdam, Meibergdreef 9, \\ 1105 AZ Amsterdam, The Netherlands \\ bLife Sciences and Health Group, Centrum Wiskunde \& Informatica (CWI), Science Park 123, 1098 \\ XG Amsterdam, The Netherlands
}

\begin{abstract}
3D dose reconstruction for radiotherapy (RT) is the estimation of the 3D radiation dose distribution patients received during RT. Big dose reconstruction data is needed to accurately model the relationship between the dose and onset of adverse effects, to ultimately gain insights and improve today's treatments. Dose reconstruction is often performed by emulating the original RT plan on a surrogate anatomy for dose estimation. This is especially essential for historically treated patients with long-term follow-up, as solely 2D radiographs were used for RT planning, and no 3D imaging was acquired for these patients. Performing dose reconstruction for a large group of patients requires a large amount of manual work, where the geometry of the original RT plan is emulated on the surrogate anatomy, by visually comparing the latter with the original 2D radiograph of the patient. This is a labor-intensive process that for practical use needs to be automated. This work presents an image-processing pipeline to automatically emulate plans on surrogate computational tomography (CT) scans. The pipeline was designed for childhood cancer survivors that historically received abdominal RT with anterior-to-posterior and posterior-to-anterior RT field set-up. First, anatomical landmarks are automatically identified on 2D radiographs. Next, these landmarks are used to derive parameters needed to finally emulate the plan on a surrogate CT. Validation was performed by an experienced RT planner, visually assessing 12 cases of automatic plan emulations. Automatic emulations were approved 11 out of 12 times. This work paves the way to effortless scaling of dose reconstruction data generation.
\end{abstract}

Keywords: Digitally reconstructed radiograph, landmark detection, radiotherapy, dose reconstruction, plan emulation

\section{INTRODUCTION}

Studies showed that cancer survivors that received radiotherapy (RT) are prone to develop late adverse effects (AEs), i.e., long-term morbidity and health problems that happen ten or twenty years after the treatment [1-3]. To improve the design of RT plans and reduce late AEs for future patients, it is important to understand how RT dose relates to late AEs. This can potentially be inferred from retrospective data. However, many cancer survivors for whom long-term follow-up is available were treated before the late-90. For these patients 3D radiation dose distributions are not available as solely 2D radiographs were used for RT planning [4]. The lack of a 3D anatomical image, i.e., computed tomography (CT), of patients precludes direct 3D dose calculation on CT as is performed routinely for currently treated patients [5]. To deal with the absence of 3D anatomical images of historically treated patients, dose reconstruction methods have been developed [6-8]. These methods provide 3D dose estimations by emulating the historical treatment on a 3D patientresembling surrogate anatomy, e.g., computational phantoms [6]. Reconstructed dose can provide crucial data to understand how deposition of radiation dose is related to AEs. However, to effectively establish such relationships, powerful data analysis techniques such as machine learning are required, involving large scale generation of dose reconstruction data. Currently, generating big amounts of reconstructed dose data in a short period of time is not possible. The challenge is not only collecting big amounts of data but is also largely due to the required time-consuming manual steps in the process.

*Further author information: (send correspondence to Z.W. or T.A.)

Z.W.: z.wang@amc.uva.nl; T.A.: t.alderliesten@amc.uva.nl 


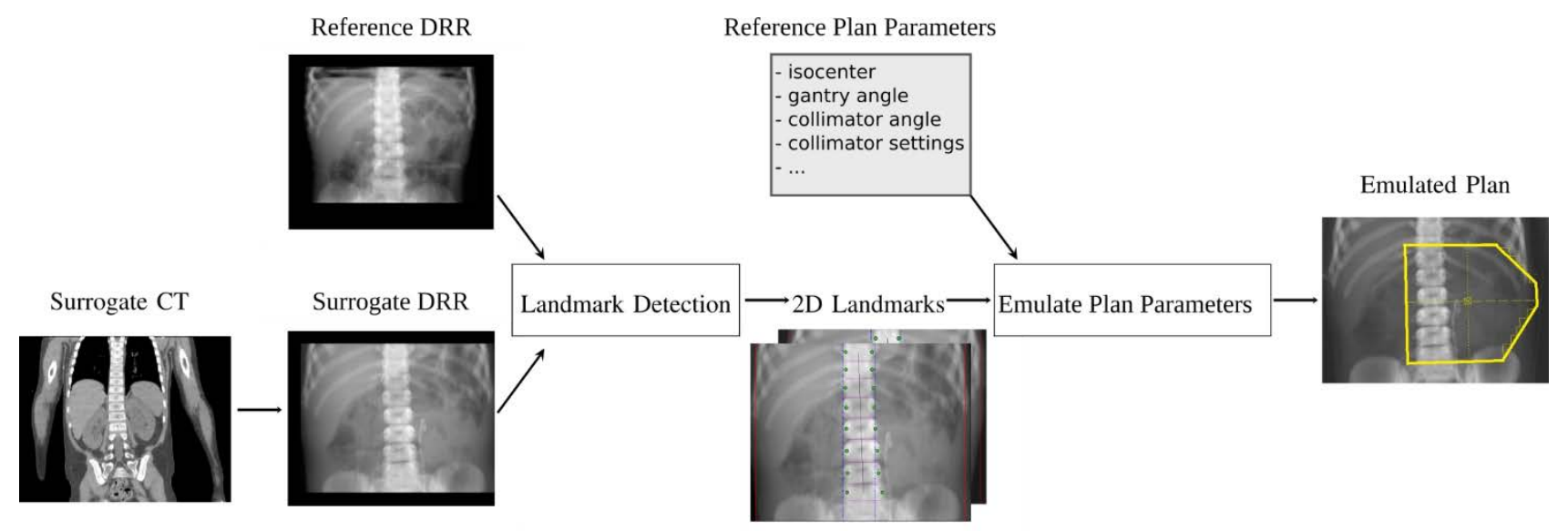

Figure 1. The proposed pipeline for automated plan emulation.

In general, dose reconstruction consists of three fundamental steps: 1. Create/select a representative surrogate anatomy; 2. Emulate the RT plan of the historical patient on the surrogate anatomy; 3. Calculate/measure the 3D dose distribution. In recent years, multiple computational phantom libraries have been developed that provide representative anatomies of patients categorized by gender, age, or even weight and height [9-11]. Studies have been published on how to match/select a surrogate phantom/CT scan that could best resemble the historical patients' anatomy [12-14]. Furthermore, the last step can also be automated, by scripting the dose calculation algorithms in a treatment planning system (TPS) or by using other out-of-field dose estimation algorithms $[8,15]$. The bottleneck that prevents performing dose reconstruction on a large scale is the intermediate step of RT plan emulation. Plan emulation is currently a manual task where an experienced RT planner makes a plan on the surrogate anatomy that resembles as much as possible the plan made for the historical patient. Reproducing the geometry of the historical plan is herein the key difficulty. The planner needs to visually check how the geometry of the RT plan on the 2D radiograph of the historical patient is configured with respect to the visible anatomy (mainly bony structures), to craft the new plan while aiming to reproduce the plan geometry on the surrogate anatomy.

In this work, we propose a pipeline of automatic plan emulation on surrogate CT scans, to enable large scale dose reconstruction. We focus on abdominal RT plans with conventional anterior-to-posterior (AP) and posterior-to-anterior (PA) field set-up (i.e., two beams irradiating from front/back of the patient body), where the geometry of the field was historically drawn on a coronal 2D radiograph of the patient. The patient group we focus on is childhood cancer patients, as they are more prone to late AEs compared to adults [2, 3], have more anatomical variations across their age range [16, 17], and dose reconstruction methods for this specific group are not well-studied [6]. The input of our pipeline is the 2D radiograph (in coronal view) of the patient for whom dose reconstruction needs to be performed (reference patient), the RT parameters of the reference plan, and the surrogate CT scan for which the plan must be emulated. The output are the RT parameters of the emulated plan, which are then used to calculate the 3D dose distribution. The pipeline of the methods is presented in Figure 1.

To enable future validation of the reconstructed dose, we use recently-treated patients' CTs and create per patient an RT record that is in line with the data available in the 2D-planning era specifically. Digitally reconstructed radiographs (DRRs) are derived from the reference CT scans, as substitutions of the historical 2D radiographs. DRRs of the surrogate CTs are also generated, as the plan will be emulated based on information extracted from DRRs. We validate our approach by asking an experienced RT planner to assess the quality of automatically emulated plans given the reference plan visualized on the reference DRR, in a blind comparison with manually emulated ones. In this pilot study, we focus on Wilms' tumor (i.e., a type of kidney tumor) plans, one of the most common abdominal cancers in children between 2 to 6 years of age.

\section{METHODOLOGY}

\subsection{Parameters of reference RT plans}

In conventional AP-PA RT planning, plan parameters that define the geometrical setup and prescribed doses of a particular plan are documented in the clinical records. In modern treatment planning systems, plan parameters are saved in a standard 
format, i.e., Digital Imaging and Communications in Medicine (DICOM) [18]. Below we list the plan parameters we consider of importance in the plan emulation process.

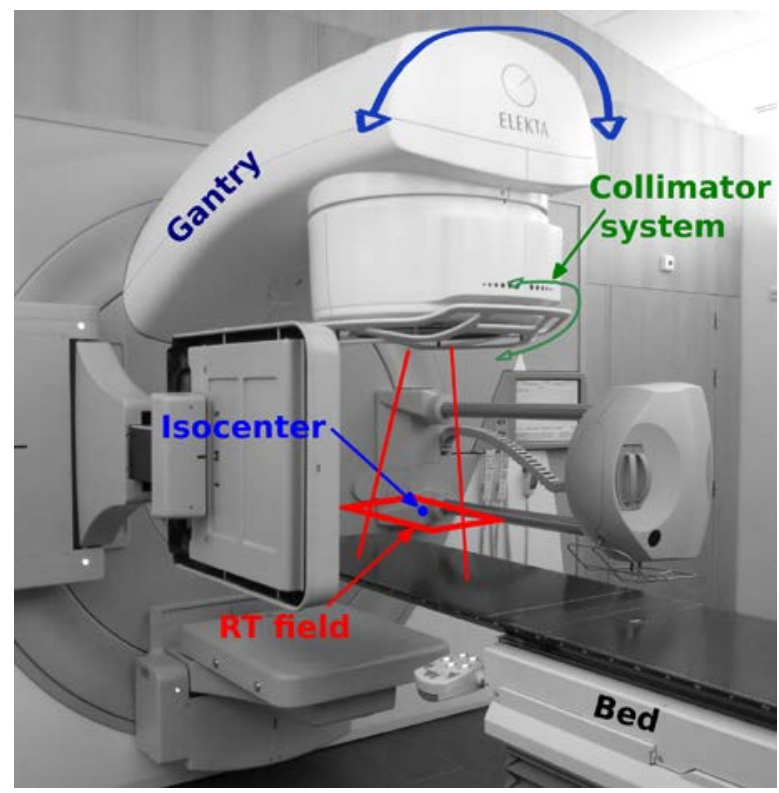

Figure 2. Illustration of components of a linear accelerator (Elekta, Crawley, UK) together with an RT field and isocenter.

The isocenter of a plan defines the point about which the collimator system and gantry of the linear accelerator (i.e., the device used for external beam RT) rotates [19] (Figure 2). The gantry angle is set as $0^{\circ}$ and $180^{\circ}$, for AP and PA beam, respectively. The collimator angle defines the orientation of the collimator coordinate system with respect to the gantry coordinate system (Figure 2). Per beam, two asymmetric jaw pairs in the collimator system are used to define the RT field size. The jaw positions are defined by the isocenter to field boundary distances in left/right and upper/lower directions in the collimator system. Multi-leaf collimators (MLC) are used to further shape the beam, to block certain structures from receiving radiation.

\subsection{Preparation of DRRs}

We use DRRs to determine the appropriate RT plan parameters. Our automatic landmark detection works on DRRs of the reference and surrogate CTs in coronal view. In order to produce DRRs of the CTs, a treatment isocenter needs to be defined since it is used to define the image plane of the DRR. However, we are here creating DRRs to emulate, among others, this RT plan parameter. Therefore, the isocenter is not yet known for the surrogate CTs. To overcome this, we use the geometrical center of the abdominal external body contour as fixed isocenter for all surrogate CT scans to create the DRRs. This is a valid approach because we found the deviations in measurements extracted from DRRs produced by use of different isocenter positions typically used for abdominal RT (e.g., left or right renal fossa, geometrical center of the body) to be negligible.

We used Oncentra TPS (version 4.3, Elekta, AB, Stockholm, Sweden) to generate the DRRs, with the following settings: window center: 3200, window level: 1441, bone threshold: 200 and bone enhancement factor: 4.

\subsection{Automatic landmark detection in DRRs}

Image processing techniques are applied on the DRRs for automatic landmark detection. An example outcome of landmark detection is displayed in Figure 3. As preprocessing steps, DRRs are automatically cropped to a common region of interest from thoracic 10th (T10) to sacral 1st (S1) vertebra, using existing delineations of the CT scans made for RT planning purposes; and a 3 by 3 pixel averaging filter is applied to suppress noise. Next, the sum of the image signal along vertical direction of the DRR is calculated. Two thresholds are applied on the summed signal to detect the horizontal boundaries of the ribs (red lines in Figure 3), and to give an initial estimation of the vertebral column (yellow lines), respectively. After that, peak detection [20], an algorithm to find the positions corresponding to local maxima in a series of signals, is 
applied on the sum of the negation (i.e., the additive inverse) of the signals along horizontal direction of the DRR within the estimated vertebral column in order to identify the intervertebral discs (pink lines). The horizontal boundaries of each vertebrae are then detected by applying peak detection on the sum of signals along vertical directions within regions defined by the estimated vertebral column (with $3 \mathrm{~mm}$ expansion on both sides) and the identified intervertebral discs. The resulting horizontal vertebrae boundaries and the vertical positions halfway of the intervertebral discs together define the middle horizontal boundaries of the vertebral bones (green dots in Figure 3).

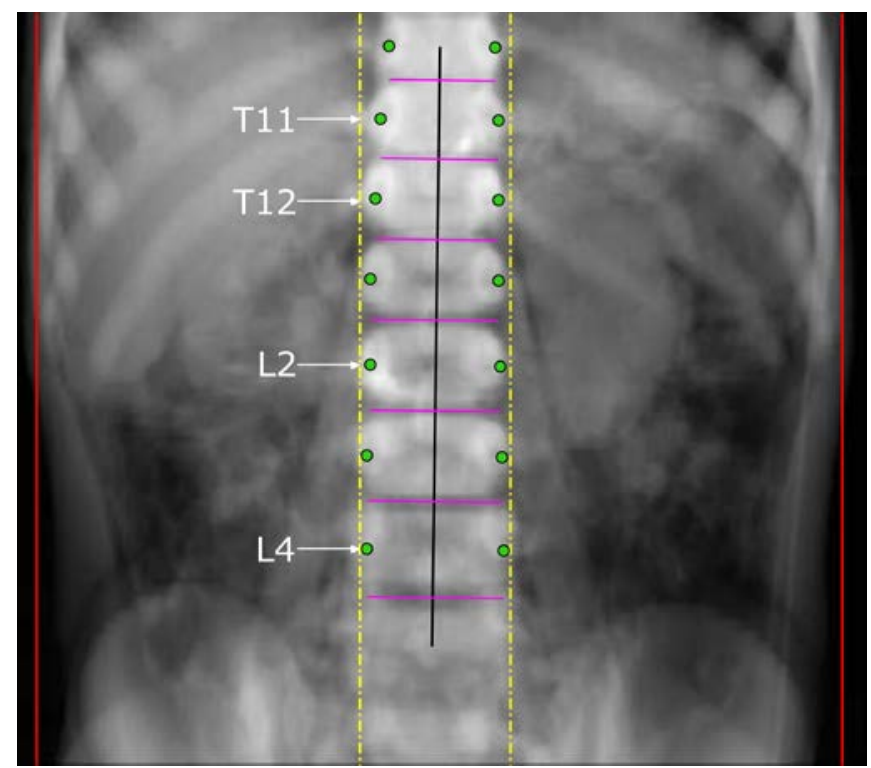

Figure 3. An example case of landmark detection in a DRR. The vertical red lines estimate the rib cage boundaries. The vertical yellow lines are the initial estimation of the vertebral column. The pink horizontal lines indicate the segments between the vertebrae bones. The green circles are middle boundaries of the vertebrae bones, and the black line fits the centers of the vertebrae, based on these identified boundaries. The T11, T12, L2, and L4 vertebrae are pointed out with arrows.

We remark that parameters for the peak detection (e.g., minimal distance between two peaks, minimal peak magnitude) are manually tuned to each specific step (but remain the same for all DRRs), to enable reliable results on all DRRs. We further estimate the vertebral column bending angle by fitting a line to the centers of the vertebrae (i.e., defined by the location in between the two identified horizontal boundaries of each vertebrae, see black line in Figure 3).

The following measures and landmarks are considered for plan emulation. Rib cage width, i.e., the distance from the leftmost point of the ribs to the right-most point of the ribs, and vertebral column length from the 11th thoracic vertebra (T11) to the 4th lumbar vertebra (L4), are considered, and are calculated based on the identified boundaries and the vertebral column bending angle. The middle-left and middle-right points on the boundaries of T12 and L2 are selected as landmarks. The coordinates of these four landmarks are mapped from the DRR to the CT coordinate system.

\subsection{Plan emulation from landmarks}

The measurements and landmark coordinates extracted from the DRRs are used to derive the parameters needed for plan emulation. In the following, $x$ refers to right-to-left (RL) direction, $z$ refers to feet-to-head (FH) direction, and $y$ refers to AP direction of the patient, in consistency with the CT coordinate system.

First, we account for dissimilarities between the reference and the surrogate DRRs in terms of bending angle, and RL and FH scaling factors, with:

$$
\begin{gathered}
\Delta \theta=0.5 \cdot\left(\theta_{\text {sur }}-\theta_{\text {ref }}\right) \\
S_{W}=\frac{\text { Wrib } b_{\text {sur }}}{W r i b_{\text {ref }}}
\end{gathered}
$$




$$
S_{L}=\frac{\text { Lvert }_{\text {sur }}}{\text { Lvert }_{\text {ref }}}
$$

where $\theta$ is the bending angle of the vertebral column, and $\Delta \theta$ the attenuated (by 0.5 ) angle difference between surrogate (sur) and reference (ref) DRR. We determined the attenuation of 0.5 after visual assessment, to avoid excessive rotations of the plan field. Wrib is the rib cage width, and Lvert is the vertebral column length from T11 to L4.

The collimator angle of the AP beam in the emulated plan can now be set to the collimator angle of the AP beam in the reference plan plus $\Delta \theta$. Similarly, $-\Delta \theta$ is used for the PA beam.

For each landmark, an estimation of the isocenter coordinates in the coronal plane for the emulated plan is given by:

$$
\left[\begin{array}{l}
x_{\text {sur }}^{i s o} \\
z_{\text {sur }}^{i s o}
\end{array}\right]=R\left(\theta_{\text {sur }}\right)\left(R\left(-\theta_{\text {ref }}\right)\left[\begin{array}{cc}
S_{W} & 0 \\
0 & S_{L}
\end{array}\right]\left[\begin{array}{c}
x_{r o f}^{\text {iso }}-x_{\text {ref }}^{l m} \\
z_{\text {ref }}^{\text {iso }}-z_{r e f}^{l m}
\end{array}\right]\right)+\left[\begin{array}{c}
x_{\text {sur }}^{l m} \\
z_{\text {sur }}^{l m}
\end{array}\right]
$$

where $\left[\begin{array}{l}x^{i s o} \\ z^{i s o}\end{array}\right]$ and $\left[\begin{array}{l}x^{l m} \\ z^{l m}\end{array}\right]$ are the coordinates of the isocenter and landmark point in the CT coordinate system, respectively, and $R(\theta)=\left[\begin{array}{cc}\cos \theta & -\sin \theta \\ \sin \theta & \cos \theta\end{array}\right]$ is the rotation matrix.

The rotation matrix is applied to correct for the influence of the bending of the vertebral column on the relative difference in location between isocenter and landmarks. Therefore, in summary, the difference in location of the isocenter with respect to the landmark is computed, scaled, and rotated to remove bending effects (terms in the brackets of Equation 4); the result is then rotated by the angle with which the surrogate vertebral column is bent compared to the reference (the left-most term $R\left(\theta_{\text {sur }}\right)$ ), and added to the coordinates of the landmark point in the surrogate DRR (right-most term).

The y coordinate of the isocenter is always set to the middle of the body contour along the AP direction, as this was standard practice in the 2D-planning era and thus used in the historically-like reference plans. We repeat the computation of the isocenter for each of the four landmarks, and finally take the average as final result, to improve the robustness of the method.

The asymmetric jaw pairs for the emulated plan are calculated as these values of the reference plan scaled by $S_{W}$ and $S_{L}$ in two directions, respectively. Similarly, the emulated MLC positions along RL are also calculated based on MLC positions of the reference plan and scaling factors $S_{W}$ and $S_{L}$.

\section{DATA}

We consider 15 abdominal CT scans of patients with age between 2 to 6 years, each including a common abdominal region from T10 to S1. The patients underwent RT between 2002 and 2018 in our institute for abdominal cancer.

We select three historical-like Wilms' tumor RT plans associated with three out of the 15 patients with two beams for left flank irradiation from AP and PA direction as reference RT plans. According to the clinical SIOP WT 2001 protocol [21], the treatment field of a typical left-sided Wilms' tumor covers the major part of the left flank with the vertebral column, the left part of the liver, and the spleen. The plans have slightly different geometry regarding the field cranial border (starting from T8, T11, or T12) and the blocked region border (rib 8, rib 10 or rib 11). For each reference plan, both manual and automatic plan emulation is applied on another four patients' CTs as surrogate CTs (i.e., in total 24 emulations are performed: 12 manual ones and 12 automated ones). 

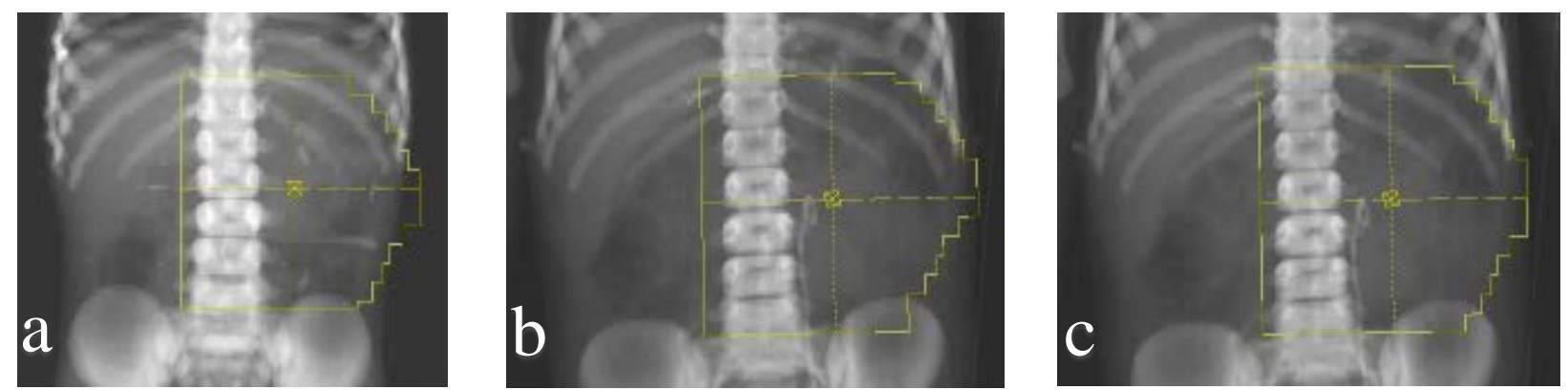

Figure 4. An example of reference and emulated plans visualized on DRRs (in coronal view). a) The reference plan on the reference DRR; b) Automatically emulated plan on the surrogate DRR; c) Manually emulated plan on the same surrogate DRR. The cross point of the two yellow dashed lines with a surrounding square indicates the isocenter position. The yellow dashed lines indicate the orthogonal coordinate axes for the collimator coordinate system. The yellow solid lines describe the effective shape of the field, where the straight lines are defined by asymmetric jaw pairs and the jagged shape are defined by MLCs. The images were generated in Oncentra TPS.
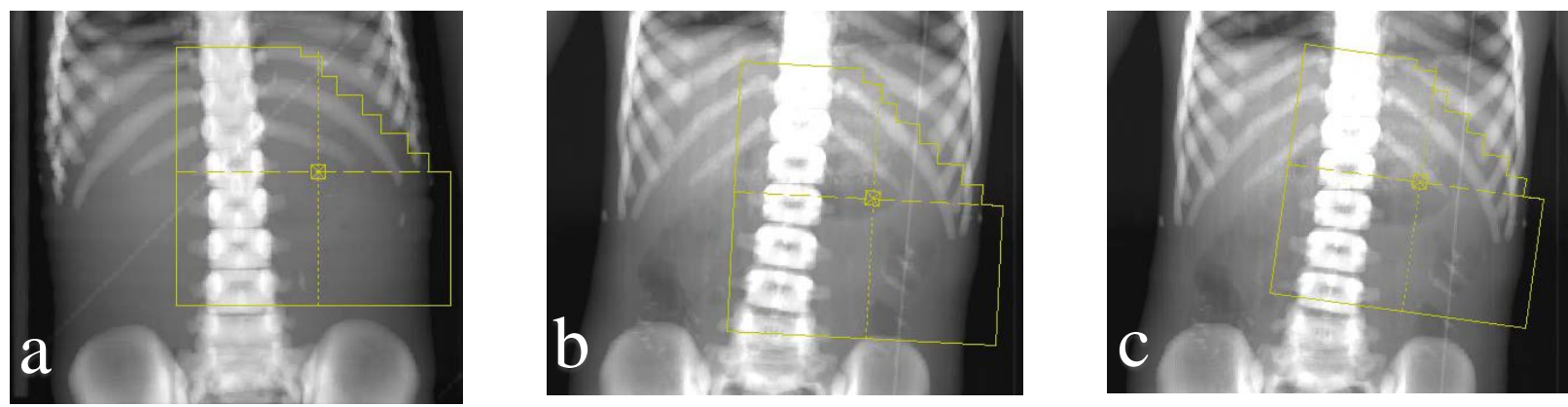

Figure 5. The failed case of reference and emulated plans visualized on DRRs (in coronal view). a) The reference plan on the reference DRR; b) Automatically emulated plan on the surrogate DRR; c) Manually emulated plan on the same surrogate DRR.

\subsection{Quality check of automatic plan emulations}

To validate our automatically emulated plans, an experienced RT planner visually assessed and compared the 24 emulations by evaluating for each of the 12 cases a reference plan on a reference CT, a manually and an automatically emulated plan on a selected surrogate CT. The plans were visualized on DRRs in Oncentra TPS. The manual plan emulation strategy is described in [22] and the resulting plans were approved by a radiation oncologist. The planner was not provided with information on which plan was manually or automatically emulated.

Further, we quantitatively compared difference in plan parameters of the automatically emulated plans compared with the manually emulated plans.

\section{RESULTS}

In Figure 4 the reference plan, the automatically emulated plan, and the manually emulated plan for one case are illustrated. The RT planner approved 11 out of 12 automatic plan emulations and 12 out of 12 manual plan emulations by visually comparing them with the reference plan. The 11 approved automatically emulated plans were considered to be of same quality as the manually emulated plans. In Figure 5 the single failed case is illustrated.

On average, the difference in isocenter location between the automatically emulated plans and the manually emulated plans was $4.4 \mathrm{~mm}$. In the failed case, the isocenter was off by $8.8 \mathrm{~mm}$ in $\mathrm{FH}$ direction and the collimator angle was off by $3^{\circ}$ of the automatically emulated plan compared to the manually emulated plan (Table 1). 
Table 1. Differences in plan parameters of the automatically emulated plans compared with the manually emulated plans for the 11 accepted cases (average value and standard deviations) and the one failed case. The Euclidean distance is used to compute difference in isocenter location.

\begin{tabular}{|l|c|c|}
\hline \multicolumn{1}{|c|}{ Plan parameters } & Average (standard deviation) & Failed case \\
\hline Isocenter location $(\mathrm{mm})$ & $4.3(3.0)$ & 3.8 \\
\hline Collimator angle $\left(^{\circ}\right)$ & $1.6(1.1)$ & 0.1 \\
\hline Field size along RL $(\mathrm{mm})$ & $2.0(1.7)$ & 4.6 \\
\hline Field size along FH $(\mathrm{mm})$ & $3.6(2.6)$ & \\
\hline
\end{tabular}

\section{DISCUSSION}

In the field of dose reconstruction, we are the first to leverage image processing techniques to automate RT plan emulation, a key component of RT dose reconstruction. With the automatic plan emulations most of the time being considered as accurate as the ones made manually on almost all of the validation comparisons, our pipeline enables large scale 3D radiation dose reconstructions in short time.

One component that is not yet fully automated is the generation of the DRRs, as we use the DRR generation module in Oncentra TPS in the data preparation step. We will further improve this by implementing DRR generation in an automatic fashion. With the help of pre-delineated organ contours, an isocenter near the $2^{\text {nd }}$ lumbar vertebra could be automatically determined. Stand-alone algorithms to calculate DRRs from CT scans are also available [23].

In this work, our pipeline, especially the landmark detection method is limited to RT plans with an AP-PA field set-up. Although only Wilms' tumor plans were assessed in this work, our approach can in principle also be effortlessly applied to different abdominal RT plans with AP-PA field set-up (e.g., neuroblastoma). Moreover, it is possible to design landmark detection methods for radiographs projecting from different gantry angles on different regions. The general idea of transforming the RT plan field based on detected landmarks can be easily extended.

There are multiple sources of uncertainty in the plan emulation pipeline. First of all, there is uncertainty from the landmark detection because the method is based on image contrast between the bone and tissue, and prior knowledge of the patient's bony anatomy is taken into account in the design of the landmark detection method. Therefore, image acquisition artifacts or abnormalities in patient anatomy (e.g., 6 lumber vertebrae instead of five) can lower the accuracy of the algorithm. In a preliminary study, a qualitative assessment of landmark detection was done by visually checking the automatically detected landmarks on the DRR (as in Figure 3), and no substantial error was observed in 43 tested cases. However, a quantitative assessment is needed to provide a more thorough validation of landmark detection. This can be achieved by asking individual RT planners to manually identify the landmarks and compare the manually labeled landmarks with the automatically labeled ones.

A second source of uncertainty is from the plan emulation step, since the transformation of the plan geometrical parameters is done under the assumption that 1) the field boundaries should be defined with respect to the rib cage width and the vertebral column length, 2) positions of the isocenter relative to the landmarks should be consistent among different patients, and 3) the vertebral column is straight (i.e., not curved), such that the collimator angle can be decided by fitting a line alongs the middle of the vertebrae. Although 1) and 2) roughly simulate the way one would manually place and adjust the plan, the variation in individual bony anatomy among patients requires consideration of a patient's anatomy as a whole, instead of just several landmark points. For example, by studying the failed case according to the RT planner's assessment (Figure 5), we found that while the reference patient's vertebral column was straight, the vertebral column of the surrogate patient was curved. This means that the constitution of the isocenter and vertebrae bones' boundaries is different from that in the reference DRR. Consequently, the automatically emulated plan had an isocenter shifted $8.8 \mathrm{~mm}$ in FH direction, a smaller field length $(4.6 \mathrm{~mm})$, and a collimator angle that differed markedly $\left(3^{\circ}\right)$ compared to the manually emulated plan. However, one could argue that in such a case, the surrogate CT is not a good representative for the reference patient's anatomy, thus it should not be selected as a surrogate CT to perform dose reconstruction.

We only tested our method for three left-sided flank RT plans on 12 surrogate CTs. In a future study, efforts will be made to improve the robustness of the pipeline and enable quality control of the automatically emulated plans. To validate the 
pipeline, a larger set of CT scans should be utilized. Further, reference plans with more variations (e.g., also including right-sided flank plans) should be considered.

\section{CONCLUSION}

In this work, we introduced a pipeline of automatic plan emulation of abdominal RT plans on surrogate CTs based on landmark detection on 2D radiographs. Our results open the possibility to automatically perform large scale of dose reconstruction data generation, for abdominal RT plans with AP-PA field set-up. In turn, this enables the use of more powerful data analysis and machine learning techniques, to study in more detail how dose causes adverse effects, giving insight into how to improve the radiation treatments of today.

\section{ACKNOWLEGEMENTS}

Financial support of this work was provided by Stichting Kinderen Kankervrij (KiKa; project no. 187). The authors further thank Koen F. Crama for his help with plan quality assessment.

\section{REFERENCES}

[1] W. D. Newhauser, A. B. de Gonzalez, R. Schulte, and C. Lee, “A Review of Radiotherapy-Induced Late Effects Research after Advanced Technology Treatments,” Front. Oncol., vol. 6, article 13, 2016.

[2] M. M. Hudson, A. C. Mertens, Y. Yasui, W. Hobbie et al., "Health status of adult long-term survivors of childhood cancer: a report from the Childhood Cancer Survivor Study,” JAMA, vol. 290, no. 12, pp. 1583-1592, 2003.

[3] Y. T. Cheung, T. M. Brinkman, C. Li, Y. Mzayek et al., "Chronic health conditions and neurocognitive function in aging survivors of childhood cancer: A report from the Childhood Cancer Survivor Study,” J. Natl. Cancer Inst., vol. 110, no. 4, pp. 411-419, 2017.

[4] D. I. Thwaites, and J. B. Tuohy, "Back to the future: the history and development of the clinical linear accelerator,” Phys. Med. Biol., vol. 51, no. 13, pp. R343, 2006.

[5] J. V. Bezin, R. S. Allodji, J.-P. Mège, G. Beldjoudi et al., “A review of uncertainties in radiotherapy dose reconstruction and their impacts on dose-response relationships,” J. Radiol. Prot., vol. 37, no. 1, pp. R1, 2017.

[6] M. Stovall, R. Weathers, C. Kasper, S. A. Smith et al., "Dose reconstruction for therapeutic and diagnostic radiation exposures: use in epidemiological studies,” Radiat. Res., vol. 166, no. 1, pp. 141-157, 2006.

[7] A. Ng, K. K. Brock, M. B. Sharpe, J. L. Moseley et al., "Individualized 3D reconstruction of normal tissue dose for patients with long-term follow-up: a step toward understanding dose risk for late toxicity," Int. J. Radiat. Oncol. Biol. Phys., vol. 84, no. 4, pp. e557-e563, 2012.

[8] C. Lee, J. W. Jung, C. Pelletier, A. Pyakuryal et al., "Reconstruction of organ dose for external radiotherapy patients in retrospective epidemiologic studies,” Phys. Med. Biol., vol. 60, no. 6, pp. 2309-2324, 2015.

[9] A. M. Geyer, S. O'Reilly, C. Lee, D. J. Long et al., "The UF/NCI family of hybrid computational phantoms representing the current US population of male and female children, adolescents, and adults-application to CT dosimetry,” Phys. Med. Biol., vol. 59, no. 18, pp. 5225, 2014.

[10] M. Stabin, M. Emmons, W. Segars, and M. Fernald, "Realistic reference adult and paediatric phantom series for internal and external dosimetry,” Radiat. Prot. Dosimetry, vol. 149, no. 1, pp. 56-59, 2011.

[11] C. Lee, J. L. Williams, C. Lee, and W. E. Bolch, "The UF series of tomographic computational phantoms of pediatric patients,” Med. Phys., vol. 32, no. 12, pp. 3537-3548, 2005.

[12] E. J. Stepusin, D. J. Long, E. L. Marshall, and W. E. Bolch, “Assessment of different patient-to-phantom matching criteria applied in Monte-Carlo based computed tomography dosimetry,” Med. Phys., vol. 44(10), pp. 5498-5508, 2017.

[13] M. Virgolin, I. W. E. M. van Dijk, J. Wiersma, C. M. Ronckers et al., "On the feasibility of automatically selecting similar patients in highly individualized radiotherapy dose reconstruction for historic data of pediatric cancer survivors,” Med. Phys., vol. 45, no. 4, pp. 1504-1517, 2018. 
[14] S. Whalen, C. Lee, J. L. Williams, and W. E. Bolch, “Anthropometric approaches and their uncertainties to assigning computational phantoms to individual patients in pediatric dosimetry studies,” Phys. Med. Biol., vol. 53, no. 2, pp. 453-471, 2008.

[15] W. Schillemans, Y. Seppenwoolde, H. Akhiat, X. Doorn et al., "SU-E-T-208: Automated Routine 3D Secondary Patient Dose Calculation Prior to and During Fractionated Treatment,” Med. Phys., vol. 40, no. 6, pp. 252, 2013.

[16] V. Varchena, “Pediatric phantoms,” Pediatr. Radiol., vol. 32, no. 4, pp. 280-284, 2002.

[17] M. J. Ackerman, “The visible human project,” Proc. IEEE, vol. 86, no. 3, pp. 504-511, 1998.

[18] P. Mildenberger, M. Eichelberg, and E. Martin, “Introduction to the DICOM standard,” Eur. Radiol., vol. 12, no. 4, pp. 920-927, 2002.

[19] R. L. Siddon, "Solution to treatment planning problems using coordinate transformations,” Med. Phys., vol. 8, no. 6, pp. 766-774, 1981.

[20] M. I. Sezan, “A peak detection algorithm and its application to histogram-based image data reduction,” Computer vision, graphics, and image processing, vol. 49, no. 1, pp. 36-51, 1990.

[21] R. Furtwängler, S. Gooskens, H. van Tinteren, J. de Kraker et al., "Clear cell sarcomas of the kidney registered on International Society of Pediatric Oncology (SIOP) 93-01 and SIOP 2001 protocols: a report of the SIOP Renal Tumour Study Group,” Eur. J. Cancer, vol. 49, no. 16, pp. 3497-3506, 2013.

[22] Z. Wang, I. W. E. M. van Dijk, J. Wiersma, C. M. Ronckers et al., “Are age and gender suitable matching criteria in organ dose reconstruction using surrogate childhood cancer patients' CT scans?,” Med. Phys., vol. 45(6), pp. 2628-2638, 2018.

[23] G. W. Sherouse, K. Novins, and E. L. Chaney, “Computation of digitally reconstructed radiographs for use in radiotherapy treatment design,” Int. J. Radiat. Oncol. Biol. Phys., vol. 18, no. 3, pp. 651-658, 1990. 